\title{
Correction to: Five non-volatile memristor enigmas solved
}

\author{
L. Chua ${ }^{1}$
}

Published online: 11 March 2019

(c) Springer-Verlag GmbH Germany, part of Springer Nature 2019

\section{Correction to: Applied Physics A (2018) 124:563 https://doi.org/10.1007/s00339-018-1971-0}

The author of the original version of this article makes the following corrections:

1. Reference 19:

R. Ascoli, L.O. Tetzlaff, J.P. Chua, R.S. Strachan, Williams, History erase effects in a non-volatile memristor. IEEE Trans. Circuits Syst. I. 63, 389-400 (2016). https:// doi.org/10.1109/TCSI.2016.2525043

Should be: A. Ascoli, R. Tetzlaff, L. O. Chua, J. P. Strachan, R. S Williams, History erase effects in a non-volatile memristor. IEEE Trans. Circuits Syst. I. 63, 389-400 (2016). https://doi.org/10.1109/TCSI.2016.2525043

2. Reference 26:

S. H. Jo, T. Chang, I. Ebong, BB. Bhadviya, P. Mazumder, W. Lu, Nanoscale memristor as synapse in neuromorphic systems. Nano Lett. 10, 1297-1301 (2010). https://doi. org/10.1021/n1904092h

Should be: S. H. Jo, T. Chang, I. Ebong, B. B. Bhadviya, P. Mazumder, W. Lu, Nanoscale memristor as synapse in neuromorphic systems. Nano Lett. 10, 1297-1301 (2010). https://doi.org/10.1021/n1904092h

3. Page 7, column 2, line 7 from bottom:

"inception" should be "inspection".

4. Page 8 , column 1 , line 8 from bottom:

"understanding this proposal" should be "understanding of this powerful new tool".

5. Page 8, column 1, line 5 from bottom:

"on exact" should be "an exact".

6. Page 13, column 1. line 2 below Sect. 4.1:

", as shown in Fig. 22." is missing after "horizontal lines".

The original article can be found online at https://doi.org/10.1007/ s00339-018-1971-0.

L. Chua

chua@berkeley.edu

1 Electrical Engineering and Computer Sciences, University

of California, Berkeley, CA 94720, USA
7. Page 13, column 2:

lines 21 and 23 from bottom: $\hat{v}$ should be $v$.

8. Page 21 , column 1 , line 10 from bottom:

"choosing of five identical" should be "choosing five identical".

9. Page 21, column 2, line 1:

"linear system which" should be "linear system theory which".

10. Page 21, column 2, line 2 from bottom:

(line 2 below Example 5.2.4):

"not periodic when" should be "not periodic over finite time when".

11. Page 29, Figure caption of Fig. 50 (column 2), line 4 from bottom:

"of the measure current" should be "of the measured current".

12. Page 35, column 2, line 9 (line 1 above Sect. 7.4):

"in Fig. 60 c, b" should be "in Fig. 60 c, d".

13. Page 40, column 2, section title 7.6.1:

"transient less" should be "transient-less".

14. Page 40, column 2, line 2 from bottom:

"xphase" should be " $x$ phase".

15. Page 41, column 2, line 9 above Sect. 7.7:

"would move the right" should be "would move to the right".

Publisher's Note Springer Nature remains neutral with regard to jurisdictional claims in published maps and institutional affiliations. 